\title{
REMARKS ON THE REPRESENTATION OF ZERO BY SOLUTIONS OF DIFFERENTIAL EQUATIONS
}

\author{
JET WIMP AND DAVID COLTON ${ }^{1}$
}

\begin{abstract}
Numerical evidence from certain problems arising in optics seems to indicate Fourier-Bessel series which converge to zero in $(1-\delta, 1]$ also converge to zero in $[1,1+\delta)$, an interval which lies outside the range of orthogonality of the Bessel functions. Here we demonstrate this as a corollary of a result on series of functions which satisfy a general SturmLiouville equation.
\end{abstract}

Sometimes it is necessary to determine the behaviour of Fourier-Bessel series

$$
\sum b_{n} J_{\nu}\left(\rho_{n} x\right), \quad \nu>-\frac{1}{2}, J_{\nu}\left(\rho_{n}\right)=0
$$

outside the interval $[0,1]$, the interval of orthogonality of the functions $J_{\nu}\left(\rho_{n} x\right)$. For instance in a certain problem concerning circularly symmetric positive filters (see [2]) it was given that the above series converged to zero for $x \in[1-\delta, 1]$. In order to guarantee a sufficient spacing in the ring pattern, it was necessary to show that it converged to zero for $x \in[1,1+\delta]$. Numerical evidence seemed to support this conclusion, and a rigorous proof of this fact has been communicated to the authors by J. Boersma, who based his analysis on the properties of Fourier-Bessel series. In this note we shall show that this result has nothing to do with any special properties (orthogonality, etc.) of Bessel functions, and is in fact true for any sequence of functions satisfying a second order differential equation of Sturm-Liouville type. This result in turn will lead to a rather surprising result on nontrivial representations of zero by infinite series of solutions to ordinary differential equations. In what follows let $\left\{c_{n}\right\}$ be any sequence of real, nonzero constants and $\left\{y_{n}\right\}$ a sequence of functions satisfying

$$
L y_{n}=c_{n}^{2} y_{n}, \quad y_{n}(0)=0, \quad y_{n}^{\prime}(0)=c_{n}
$$

where $L=-d^{2} / d x^{2}+q(x)$ and $q \in C[-\delta, \delta]$. Note that in the analysis which follows the initial condition

$$
y_{n}^{\prime}(0)=c_{n}
$$

Received by the editors May 1, 1978.

AMS (MOS) subject classifications (1970). Primary 42A52; Secondary 33A40, 34B25.

Key words and phrases. Fourier-Bessel series, uniqueness sets, representations of zero, orthogonal functions.

${ }^{1}$ The research of this author was supported in part by AFOSR Grant 76-2879 and NSF Grant MCS77-02056. 
merely plays the role of a normalization constant.

THEOREM. If $\Sigma\left|a_{n}\right|<\infty$ and

$$
0=\sum a_{n} y_{n}(x), \quad x \in[0, \delta]
$$

then

$$
0=\sum a_{n} y_{n}(x), \quad x \in[-\delta, 0] .
$$

Proof. We use the representation [1, Appendix IV],

$$
y_{n}(x)=\sin \left(c_{n} x\right)+\int_{0}^{x} K(x, t) \sin \left(c_{n} t\right) d t, \quad x \in[-\delta, \delta]
$$

where $K(x, t)$ is a continuously differentiable function of $x$ and $t$ that is uniquely determined by the function $q$ and the condition $K(0,0)=0$. Since $K$ is continuous and $\left|a_{n} y_{n}(x)\right| \leqslant M\left|a_{n}\right|$,

$$
M=1+\sup _{x} \int_{0}^{x}|K(x, t)| d t, \quad x \in[-\delta, \delta],
$$

the series (1) and (2) converge absolutely and uniformly for $x \in[-\delta, \delta]$. Multiplying (3) by $a_{n}$ and summing shows that

$$
0=u(x)+\int_{0}^{x} K(x, t) u(t) d t, \quad x \in[0, \delta], u(x)=\sum a_{n} \sin \left(c_{n} x\right)
$$

and by the uniqueness of solutions to Volterra integral equations this means

$$
u(x)=0, \quad x \in[0, \delta] \text {. }
$$

Furthermore

$$
y_{n}(-x)=-\sin \left(c_{n} x\right)+\int_{0}^{x} K(-x,-t) \sin \left(c_{n} t\right) d t, \quad x \in[0, \delta]
$$

so

$$
\sum a_{n} y_{n}(-x)=-u(x)+\int_{0}^{x} K(-x,-t) u(t) d t=0, \quad x \in[0, \delta] .
$$

This concludes the proof of the Theorem.

Returning now to our Fourier-Bessel series we note that $y_{n}(x)=$ $x^{1 / 2} J_{\nu}\left(\rho_{n} x\right)$ satisfies the equation

$$
L y_{n}=\rho_{n}^{2} y_{n}
$$

with $q(x)=x^{-2}\left(\nu^{2}-\frac{1}{4}\right)$. Hence replacing $x$ by $1-x, c_{n}$ by $\rho_{n}$, and using well-known asymptotic properties of Bessel functions we have

COROLlaRY 1. Let $\sum n^{-1 / 2}\left|b_{n}\right|<\infty$,

$$
\sum b_{n} J_{\nu}\left(\rho_{n} x\right)=0, \quad x \in[1-\delta, 1] \text {. }
$$

Then the same holds for $x \in[1,1+\delta]$.

From the proof of the Theorem we arrive at the surprising 
Corollary 2. Let $\Sigma\left|a_{n}\right|<\infty$. Then

$$
0=\sum a_{n} y_{n}(x), \quad x \in[0, \delta]
$$

if and only if

$$
0=\sum a_{n} \sin \left(c_{n} x\right), \quad x \in[0, \delta] .
$$

In other words a nontrivial representation of zero on an interval in terms of one set of functions leads to another nontrivial representation of zero with the same coefficients but different functions in the expansion. One's first reaction is to suspect that all the coefficients $a_{n}$ must be zero if $\Sigma\left|a_{n}\right|<\infty$ and (4) or (5) is true. But it is quickly seen that this need not be the case if one considers a function $f \in C^{2}[0, \pi], f \neq 0$, such that $f(\pi)=0, f(x)=0$ for $x \in[0, \delta]$ and expands $f$ in a Fourier sine series. In this case we have that $a_{n} \neq 0$ for all $n$, $\left|a_{n}\right|=O\left(n^{-2}\right)$, and (5) is valid for $c_{n}=n$. It can also be shown that if $f \in C^{k}[0,1], f^{(j)}(1)=0,0 \leqslant j \leqslant k-1$, then the Fourier-Bessel coefficients of $f$ are $O\left(n^{1-k}\right)$. Thus if $k \geqslant 2$ and we make $f(x)>0$ on $[0,1-\delta]$ and zero on $[1-\delta, 1]$ then $\Sigma n^{-1 / 2}\left|b_{n}\right|<\infty$ (see Corollary 1) but clearly $b_{n}$ will not be zero for all $n$.

It would be interesting to determine necessary conditions on the sequence $\left\{c_{n}\right\}$ such that $\Sigma\left|a_{n}\right|<\infty$ and (5), or equivalently (4), imply that all the $a_{n}$ are zero.

\section{REFERENCES}

1. B. Ja. Levin, Distribution of zeros of entire functions, Transl. Math. Monographs, vol. 5, Amer. Math. Soc., Providence, R. I., 1964, Theorem 1, p. 427.

2. P. Wiersma, The realization of circularly symmetric positive filter functions by transparent rings, Technical Report, Department of Electrical Engineering, Eindhoven University of Technology, Eindhoven, The Netherlands, 1978.

Department of Mathematics, Drexel University, Philadelphia, Pennsylvania 19104

Department of Mathematics, University of Delaware, Newark, Delaware 19711 